PROCEEDINGS OF THE

AMERICAN MATHEMATICAL SOCIETY

Volume 133, Number 3, Pages 681-686

S 0002-9939(04)07574-

Article electronically published on August 24, 2004

\title{
SIMPLICITY OF NONCOMMUTATIVE DEDEKIND DOMAINS
}

\author{
K. R. GOODEARL AND J. T. STAFFORD
}

(Communicated by Lance W. Small)

\begin{abstract}
The following dichotomy is established: A finitely generated, complex Dedekind domain that is not commutative is a simple ring. Weaker versions of this dichotomy are proved for Dedekind prime rings and hereditary noetherian prime rings.
\end{abstract}

\section{INTRODUCTION}

When the classical concept of a Dedekind domain was extended to noncommutative rings, the natural examples that arose were either classical orders (and hence finitely generated modules over their centres) or simple rings such as the Weyl algebra $A_{1}(\mathbb{C})$. Indeed, among finitely generated Dedekind domains over algebraically closed fields, classical orders and simple rings are the only known examples. This dichotomy in the examples suggests that an actual dichotomy might exist among general Dedekind domains, although we are not aware that any such conjecture has been formulated in the literature. The main goal in this paper is to establish just such a result as well as to give similar dichotomies for Dedekind prime rings and HNP rings.

Before stating the first theorem we need some definitions. An HNP ring is simply a (nonartinian) hereditary noetherian prime ring, while a Dedekind prime ring is an HNP ring for which each nonzero ideal $I$ is invertible in the sense that there exists a subbimodule $I^{-1}$ of the Goldie quotient ring $Q=Q(R)$ of $R$ such that $I I^{-1}=I^{-1} I=R$. Various equivalent definitions are given in [MR, $\left.\S 5.2\right]$.

In fact the dichotomy is stronger than the one suggested above:

Theorem 1. Let $R$ be a Dedekind domain that is a finitely generated algebra over an uncountable, algebraically closed field $k$. Then $R$ is either simple or commutative.

The finite generation assumption can be further weakened to the assertion that $\operatorname{dim}_{k} R<\operatorname{card} k$ (see Theorem 6 ), but some such assumption is clearly necessary. Indeed, in the opposite direction one has the following result from [GS]: Suppose that $R$ is a noncommutative UFD in the sense that $R$ is a noetherian domain for which each height-one prime ideal $P$ satisfies (i) there exists $p \in P$ with $P=$

Received by the editors November 6, 2003.

2000 Mathematics Subject Classification. Primary 16P40, $16 \mathrm{E} 60$.

Key words and phrases. Dedekind domain, simple ring, invertible ideal, HNP ring.

The research of both authors was partially supported by grants from the National Science Foundation. Some of it was carried out while the authors participated in the Noncommutative Algebra Year (1999-2000) at the Mathematical Sciences Research Institute in Berkeley, and they thank MSRI for its support.

(C)2004 American Mathematical Society 
$p R=R p$ and (ii) $R / P$ is a domain. These exist in profusion; for example, take the enveloping algebra of any finite-dimensional complex nilpotent Lie algebra. The set $\mathcal{C}=\bigcap\{R \backslash P\}$, where $P$ runs through the height-one prime ideals of $R$, is localizable in $R$. If $R$ is not commutative, then the localization $T=R_{\mathcal{C}}$ is necessarily a principal ideal domain and hence a Dedekind domain [GS, Corollary 1]. However, each height-one prime ideal of $R$ induces a height-one prime ideal of $T$.

We give a number of variants of Theorem 1. For example, an HNP domain $R$ that is finitely generated over an uncountable algebraically closed field $k$ either satisfies a polynomial identity (PI) or has no proper invertible ideals. To help place this in context, recall that an HNP ring $R$ is Dedekind prime if and only if it has no nonzero proper idempotent ideals [MR, Proposition 5.6.3].

The key to proving all these results rests upon the following result that neither requires $R$ to be HNP nor requires any hypotheses on $k$.

Theorem 2. Let $R$ be a prime noetherian algebra over a field $k$ and $N$ a proper invertible ideal of $R$. If $\operatorname{dim}_{k}(R / N)<\infty$, then $R$ is a PI ring.

\section{Proof of Theorems 1 AND 2}

We first prove Theorem 2 , for which we need some notation. If $J$ is an ideal in a ring $R$, write $\mathcal{C}_{R}(J)$ for the set of elements of $R$ that become regular modulo $J$. The ideal $J$ is said to be (right and left) localizable if $\mathcal{C}_{R}(J)$ is a (right and left) denominator set in $R$, in which case the localization $R\left[\mathcal{C}_{R}(J)^{-1}\right]$ is denoted $R_{J}$.

Proof of Theorem 2. Let $J=\sqrt{N}$ denote the radical of $N$. By Ja Proposition 3.3.18], $N$ has the (right and left) Artin-Rees property. Since $J^{r} \subseteq N$, for some $r$, it follows that $J$ also has the Artin-Rees property. For any $n \geq 1$, the ring $R / J^{n}$ is artinian and so $\mathcal{C}_{R / J^{n}}\left(J / J^{n}\right)$ is just the set of units in $R / J^{n}$. Thus the hypotheses of [MR, Proposition 4.2.10] are satisfied and, by that result, $J$ is (right and left) localizable. By [Ja, Theorem 3.2.3], the localization $S=R_{N}$ has Jacobson radical $J(S)=J S=S J$. Also, $S / N S \cong R / N$.

The $\mathcal{C}_{R}(J)$-torsion submodule of $R_{R}$ is an ideal $I$ that contains no regular elements. Since $R$ is prime, this forces $I=0$. The same result holds on the left and so $\mathcal{C}_{R}(J)$ consists of regular elements. Thus $S$ can be identified with a prime noetherian subring of $Q=Q(R)$. Now $S N^{-1} S$ is an $S$-subbimodule of $Q$ for which $\left(S N^{-1} S\right)(N S)=S=(N S)\left(S N^{-1} S\right)$. Thus, $N S$ is an invertible ideal of $S$. It suffices to show that $S$ is PI and so, replacing $R$ by $S$, we may assume that $R$ is semilocal with $J(R)=\sqrt{N}$.

Pick a regular element $a \in N$. By [Le, Theorem 2.4], $R$ has (Rentschler-Gabriel) Krull dimension one, and so $R / a R$ has finite length. Hence $a R \supseteq J(R)^{s} \supseteq N^{s}$, for some $s$. Since $R / N$ is finite dimensional, so are $R / N^{s}$ and $R / a R$. On the other hand, $J(R)^{t} \neq J(R)^{t+1}$ for any $t$ and so $\bigcap_{n \geq 0} a^{n} R \subseteq \bigcap_{n \geq 0} J(R)^{n}=0$. Thus [FS. Lemma 1.1] implies that $R$ satisfies the standard identity $s_{2 d}$ for $d=$ $\operatorname{dim}_{k}(R / a R)$.

The following weak version of Theorem 1 is an easy consequence of Theorem 2 . A field $F$ is called separably closed if it has no finite-dimensional separable extensions. The algebraic closure of a field $F$ will always be written $\bar{F}$. The assumptions of the next result are rather artificial since we will subsequently need two particular cases of it. 
Corollary 3. Let $R$ be an algebra over a separably closed field $k$, such that $\operatorname{dim}_{k} R<$ card $k$ and $R \otimes_{k} \bar{k}$ is noetherian. Then:

(1) If $R$ is HNP, then either $R$ is a PI ring or $R$ has no proper invertible ideals.

(2) If $R$ is a Dedekind prime ring, then $R$ is either PI or simple.

Proof. (1) Assume that $R$ has proper invertible ideals, and let $N$ be a maximal one. Then $N$ is semiprime [ER, Theorem 2.6], and so $R / N \cong \bigoplus_{i=1}^{t} M_{n_{i}}\left(D_{i}\right)$ for some $n_{i}$ and division $k$-algebras $D_{i}$. Since $\operatorname{dim}_{k} D_{i} \leq \operatorname{dim}_{k} R<\operatorname{card} k$, [Pa Lemma 7.1.2] implies that each $D_{i}$ is algebraic over $k$. Suppose that $D_{i}$ is not commutative and let $Z_{i}=Z\left(D_{i}\right)$ denote the centre of $D_{i}$. By the Koethe-Noether-Jacobson Theorem Rw, Exercise 2.8, p.312] there exists $f \in D_{i} \backslash Z_{i}$ such that $f$ is separable over $Z_{i}$. Since $k$ is separably closed, $Z_{i}(f) / k$ and hence $Z_{i}(f) / Z_{i}$ is purely inseparable, giving a contradiction. Thus each $D_{i}$ is commutative.

Now $(R / N) \otimes_{k} \bar{k}$ is noetherian and hence so is each $D_{i} \otimes_{k} \bar{k}$. Since $D_{i} \subseteq \bar{k}$, faithful flatness implies that $D_{i} \otimes_{k} D_{i}$ is noetherian, which by [Va, Theorem 11] implies that $\operatorname{dim}_{k} D_{i}<\infty$. Thus Theorem 2 implies that $R$ is PI.

(2) If $R$ is not simple, then it has a proper invertible ideal and so part (1) applies.

In order to obtain Theorem 1, we need to understand PI algebras satisfying the hypotheses of the corollary.

Lemma 4. Let $R$ be a PI ring over an algebraically closed field $k$, such that $\operatorname{dim}_{k} R<\operatorname{card} k$. If $R$ is an HNP domain, then $R$ must be commutative.

Proof. By [MR, Theorem 13.9.16 and Definition 5.3.5], the centre $Z=Z(R)$ is a Dedekind domain and $R$ is a finitely generated $Z$-module. Assume for the moment that $Z$ has transcendence degree at most one over $k$ and let $F$ denote the field of fractions of $Z$. Then the Brauer group of $F$ is trivial by Tsen's Theorem $\mathrm{Pi}$. Corollary a, p.376]. Since $Q=Q(R)$ is a central simple algebra with centre $F$, this forces $Q \cong M_{n}(F)$ for some $n$. But $Q$ is a division ring, so $n=1$ and $Q=F$. Thus $Q$ and $R$ are commutative.

Thus, it remains to prove:

Sublemma 5. Let $Z$ be a commutative noetherian domain of Krull dimension one that is an algebra over an algebraically closed field $k$ with $\aleph=\operatorname{dim}_{k} Z<\operatorname{card} k=\beth$. Then $Z$ has transcendence degree at most one over $k$.

Proof. If the sublemma fails, pick a polynomial subring $k[x, y] \subseteq Z$. We will need the following observation.

(†) Let $F[w]$ be a polynomial extension of a field $F$. If $\lambda_{i}$, for $i \in I$, are distinct elements of $F$, then the set $\left\{\left(w-\lambda_{i}\right)^{-1}: i \in I\right\} \subset F(w)$ is linearly independent over $F$. (See, for example, the proof of [Pa, Lemma 7.1.2].)

By $(\dagger)$, the set $I=\{\lambda \in k:(x-\lambda) Z \neq Z\}$ has cardinality $\beth$. If $\lambda \in I$, then $\bar{Z}=$ $Z /(x-\lambda) Z$ is a nonzero artinian ring. By [Pa, Lemma 7.1.2], again, each factor field of $\bar{Z}$ is equal to $k$ and so $\bar{Z}$ is finite dimensional over $k$. This implies that the powers of $y$ become linearly dependent in $\bar{Z}$ and so there exists $f_{\lambda} \in k[y]^{*}=k[y] \backslash\{0\}$ such that $f_{\lambda} \in(x-\lambda) Z$. In particular, $z_{\lambda}=(x-\lambda)^{-1} f_{\lambda} \in Z$. Let $Z(y) \subseteq Q(Z)$ denote the localization of $Z$ at $k[y]^{*}$. Then (†) implies that $\left\{z_{\lambda}: \lambda \in I\right\}$ is linearly independent over $k(y)$. On the other hand, $\operatorname{dim}_{k(y)} Z(y) \leq \aleph<\beth=\operatorname{card} I$, giving the required contradiction. This completes the proof of both Sublemma 5 and Lemma 4. 
We note that Lemma 4 also holds if the hypothesis " $\operatorname{dim}_{k} R<\operatorname{card} k$ " is replaced by " $R$ is a finitely generated $k$-algebra". The proof, which uses the Artin-Tate Lemma, is left to the interested reader.

Combining Lemma 4 with Corollary 3 gives the following generalization of Theorem 1.

Theorem 6. Let $R$ be a domain over an algebraically closed field $k$, such that $\operatorname{dim}_{k} R<\operatorname{card} k$. Then:

(1) If $R$ is HNP but not commutative, then $R$ has no proper invertible ideals.

(2) If $R$ is a Dedekind domain, then $R$ is either commutative or simple.

Corollary 7. Let $R$ be a Dedekind prime ring over an algebraically closed field $k$, such that $\operatorname{dim}_{k} R<\operatorname{card} k$. Then $R$ is either simple or Morita equivalent to a commutative domain.

Proof. By [MR, Proposition 5.2.12], $R$ is Morita equivalent to a Dedekind domain.

There is one dichotomy in the literature, due to L. W. Small, with a flavour similar to that of Theorem 6: A prime, noetherian, finitely generated complex algebra of Krull dimension one is either primitive or satisfies a polynomial identity [FS, p.251].

\section{DEDEKIND PRIME ALGEBRAS OVER NON-ALGEBRAICALLY CLOSED FIELDS}

Theorem 1 obviously fails if we remove the hypothesis that the base field be algebraically closed - just consider the principal ideal domain $\mathbb{H}[x]$, viewed as an algebra over $\mathbb{R}$. However, this algebra is PI, which suggests that a version of Corollary 3 might still hold. We prove one such result in this section.

Lemma 8. Let $R$ be a semihereditary algebra over a field $k$. If $F \supseteq k$ is a separable algebraic field extension, then $R \otimes_{k} F$ is semihereditary.

Proof. Since $R \otimes_{k} F$ is a directed union of subalgebras $R \otimes_{k} F^{\prime}$ where $F^{\prime} \supseteq k$ is a finite separable field extension, we may assume that $F$ is finite over $k$. Then by [Pa, Lemma 7.2.3], the algebra $S=R \otimes_{k} F$ is relatively projective with respect to $R$, in the sense that any short exact sequence of $S$-modules that splits as a sequence of $R$-modules also splits as a sequence of $S$-modules.

Now consider a finitely generated right ideal $I$ of $S$ and write $I \cong S^{n} / K$ for some $S$-submodule $K$ of the free $S$-module $S^{n}$. Since $S_{R}$ is free and $I_{R}$ is finitely generated, $I_{R}$ must be projective, and so $K$ is an $R$-module direct summand of $S^{n}$. By relative projectivity, $K$ is also an $S$-module direct summand of $S^{n}$, whence $I_{S}$ is projective. Therefore $S$ is right semihereditary. By symmetry, it is also left semihereditary.

Theorem 9. Let $R$ be an algebra over a field $k$ such that $R \otimes_{k} \bar{k}$ is noetherian and $\operatorname{dim}_{k} R<\operatorname{card} k$. Then:

(1) If $R$ is HNP, then either $R$ is a PI ring or $R$ has no proper invertible ideals.

(2) If $R$ is a Dedekind prime ring, then $R$ is either PI or simple.

Proof. Part (2) follows immediately from part (1), so assume that $R$ is HNP. If $\widetilde{k}$ denotes the separable closure of $k$, then faithful flatness implies that $\widetilde{R}=R \otimes_{k} \widetilde{k}$ is also noetherian. By Lemma $8, \widetilde{R}$ is semihereditary and therefore hereditary. 
Moreover, $\widetilde{R}$ is semiprime by [Ya, Proposition 1.12]. We identify $R$ with the $k$ subalgebra $R \otimes 1 \subseteq \widetilde{R}$.

By [MR, Theorem 5.4.6], $\widetilde{R}=R_{0} \oplus \cdots \oplus R_{n}$ where $R_{0}$ is artinian and $R_{1}, \ldots, R_{n}$ are HNP. Since $\widetilde{R}$ is semiprime, $R_{0}$ must be semisimple. The regular elements of $R$ remain regular in $\widetilde{R}$, and they form a denominator set in that ring. Hence, there are natural inclusions $\widetilde{R} \subseteq Q \otimes_{k} \widetilde{k} \subseteq R_{0} \oplus Q_{1} \oplus \cdots \oplus Q_{n}$ where $Q=Q(R)$ and $Q_{\ell}=Q\left(R_{\ell}\right)$ for $\ell \geq 1$. Note that

$$
\operatorname{dim}_{\tilde{k}} R_{\ell} \leq \operatorname{dim}_{k} R<\operatorname{card} k \leq \operatorname{card} \widetilde{k}
$$

and so Corollary 3 applies to $R_{\ell}$ for $\ell \geq 1$.

Suppose that $R$ has a proper invertible ideal $I$. Then $I$ induces an ideal $J=$ $\bigoplus_{\ell=0}^{n} J_{\ell} \subseteq \widetilde{R}$, and $I^{-1}$ induces an $\widetilde{R}$-subbimodule $J^{\prime} \subseteq Q \otimes_{k} \widetilde{k}$ such that $J J^{\prime}=$ $J^{\prime} J=\widetilde{R}$. It follows that each $J_{\ell}$ is an invertible ideal in $R_{\ell}$. Moreover, using [MR, (5.6.2)], $\bigcap_{j>0} J_{\ell}^{j} \subseteq \bigcap_{j>0}(I \otimes \widetilde{k})^{j}=0$ for each $\ell$. If $\ell=0$, the first of these observations implies that $J_{0}=R_{0}$, and the second then implies that $R_{0}=0$.

On the other hand, $J_{\ell}$ is a proper invertible ideal in $R_{\ell}$ for $\ell \geq 1$. Clearly $R_{\ell} \otimes_{\tilde{k}} \bar{k}$ is a summand of $\widetilde{R} \otimes_{\tilde{k}} \bar{k}=R \otimes_{k} \bar{k}$ and so $R_{\ell} \otimes_{\tilde{k}} \bar{k}$ is noetherian. Thus the hypotheses of Corollary 3 are satisfied and each $R_{\ell}$ is PI. Therefore $R$ is PI.

As we mentioned in the introduction, the examples from [GS] show that the theorem fails badly if $\operatorname{dim}_{k} R=$ card $k$. Similarly it can fail badly if $R \otimes_{k} \bar{k}$ is not noetherian. Here is a typical example. Let $D$ denote the Krull division ring; thus $D=\bigotimes_{\mathbb{Q}} D_{p}$, where for each prime $p \in \mathbb{Z}, D_{p}$ is a $p^{2}$-dimensional, $\mathbb{Q}$-central division ring (see, for example, [SW1, p.221]). Let $k=\mathbb{Q}\left(x_{i}: i \in I\right)$ be a purely transcendental field extension of $\mathbb{Q}$ of cardinality $\geq \aleph_{1}$. Then, $E=D \otimes_{\mathbb{Q}} k$ is a division ring and $R=E[y]$ is a Dedekind domain for which the conclusion of Theorem 9 fails. Indeed, for each prime $p$ there exists $f_{p} \in \mathbb{Q}[y]$ such that $R / f_{p} R \cong$ $M_{p}\left(F_{p}\right)$, for the appropriate division ring $F_{p}$ algebraic over $k$. Thus, $R$ is not PI but, of course, each $f_{p} R$ is an invertible ideal and $\operatorname{dim}_{k} R=\aleph_{0}<$ card $k$. Examples of other HNP rings with distinctive ideal structures can be found in [SW1] and [SW2]. To give one example, let $H$ be the ring constructed in [SW2, Theorem 2.2]. Then $S=H \otimes_{\mathbb{Q}} k$ will be a subring of $R$ such that $S$ is an HNP ring with infinitely many idempotent ideals.

The first example from the last paragraph also shows that Theorem 2 will fail if we weaken the assumption that $\operatorname{dim}_{k}(R / N)<\infty$ to " $\operatorname{dim}_{k}(R / N)<\operatorname{card} k$ " or even to " $R / N$ is algebraic over the uncountable field $k$."

\section{REFERENCES}

[ER] D. Eisenbud and J. C. Robson, Hereditary noetherian prime rings, J. Algebra 16 (1970), 86-104. MR 0291222 (45:316)

[FS] D. R. Farkas and L. W. Small, Algebras which are nearly finite dimensional and their identities, Israel J. Math. 127 (2002), 245-251. MR 1900701 (2003c:16033)

[GS] M. Gilchrist and M. Smith, Noncommutative UFDs are often PIDs, Math. Proc. Cambridge Phil. Soc. 96 (1984), 417-419. MR 0755829 (85h:16002)

[Ja] A. V. Jategaonkar, Localization in Noetherian Rings, London Math. Soc. Lecture Note Series 98, Cambridge University Press, Cambridge, 1986. MR 0839644 (88c:16005)

[Le] T. H. Lenagan, Krull dimension and invertible ideals in noetherian rings, Proc. Edinburgh Math. Soc. 20 (1976), 81-86. MR 0419520 (54:7541) 
[MR] J. C. McConnell and J. C. Robson, Noncommutative Noetherian Rings, Wiley-Interscience, New York, 1987; Revised Edition, Amer. Math. Soc., Providence, 2001. MR 1811901 (2001i:16039)

[Pa] D. S. Passman, The Algebraic Structure of Group Rings, Wiley, New York, 1977; Reprinted Edition, Krieger, Malabar, FL, 1985. MR 0470211 (81d:16001)

[Pi] R. S. Pierce, Associative Algebras, Graduate Texts in Math. 88, Springer-Verlag, New York, 1982. MR 0674652 (84c:16001)

$[\mathrm{Rw}]$ L. H. Rowen, Ring Theory, Vol. I, Academic Press, San Diego, 1988. MR 0940245 (89h:16001)

[SW1] J. T. Stafford and R. B. Warfield, Jr., Hereditary orders with infinitely many idempotent ideals, J. Pure Appl. Algebra 31 (1984), 217-225. MR 0738216 (86c:16002)

[SW2] J. T. Stafford and R. B. Warfield, Jr., Construction of hereditary noetherian rings and simple rings, Proc. London Math. Soc. 51 (1985), 1-20. MR 0788847 (86j:16016)

[Va] P. Vámos, On the minimal prime ideals in a tensor product of fields, Math. Proc. Cambridge Phil. Soc. 84 (1978), 25-35. MR 0489566 (80j:12016)

[Ya] S. Yammine, Les théorèmes de Cohen-Seidenberg en algèbre non commutative, in Séminaire d'Algèbre Paul Dubreil 1977-78 (M.-P. Malliavin, Ed.), Lecture Notes in Math. 740, Springer-Verlag, Berlin, 1979, pp. 120-169. MR 0563499 (81i:16004)

Department of Mathematics, University of California, Santa Barbara, California 93106-3080

E-mail address: goodearl@math.ucsb.edu

Department of Mathematics, University of Michigan, Ann Arbor, Michigan 481091109

E-mail address: jts@umich.edu 